\title{
Streptococcal Pharyngitis: Delving Deeper than the Throat
}

\author{
Sadaf Sheikh ${ }^{1}$, Umair Javed ${ }^{2}$ and Muhammad Akbar Baig ${ }^{2}$ \\ ${ }^{1}$ Department of Emergency Medicine, Sultan Qaboos University Hospital, Muscat, Oman \\ ${ }^{2}$ Department of Emergency Medicine, Aga Khan University Hospital, Karachi, Pakistan
}

\begin{abstract}
Acute rhabdomyolysis and elevated transaminases during streptococcal pharyngitis are rare presentations. The proposed pathophysiological mechanisms include direct bacterial invasion and toxin generation. Physicians should be aware of the association between these infections and the above-mentioned complications to facilitate optimal treatment of these patients. We present the case of a 18-years-old gentleman with $\beta$-haemolytic streptococcal pharyngitis complicated by rhabdomyolysis and elevated liver function tests. Such high levels of creatine phosphokinase of $111856 \mathrm{IU} / \mathrm{L}$ and elevated liver function tests with aspartate aminotransferase (AST) of $1862 \mathrm{U} / \mathrm{L}$ and alanine aminotransferase (ALT) of $1003 \mathrm{U} / \mathrm{L}$ in streptococcal pharyngitis is rare to find in the literature. He was treated with aggressive intravenous hydration, antibiotics and hemodialysis.
\end{abstract}

Key Words: Rhabdomyolysis, $\beta$-haemolytic Streptococci, Creatine phosphokinase, Liver function tests.

How to cite this article: Sheikh S, Javed U, Baig MA. Streptococcal Pharyngitis: Delving Deeper than the Throat. J Coll Physicians Surg Pak 2021; 31(06):732-734.

\section{INTRODUCTION}

$\beta$-haemolytic streptococci infections can manifest as pharyngitis, cellulitis, primary bacteremia, arthritis, endocarditis, meningitis, pneumonia, necrotizing fasciitis, myositis, and even fatal toxic shock-like syndrome. ${ }^{1}$ Pathophysiological mechanismlinking $\beta$-haemolyticstreptococci-related pharyngitis and rhabdomyolysis yet remain unclear. ${ }^{2}$ We, herein, present a case of an 18-year gentleman with rhabdomyolysis and raised transaminases. The clinical presentation showed unrelenting muscle pain, concentrated urine output and fever. $\beta$-haemolytic streptococci-related upper respiratory tract infection with raised anti-streptolysin (ASO) and creatine phosphokinase (CPK) is very suggestive of rhabdomyolysis as a complication of streptococcal pharyngitis. Deranged kidney function and raised urine myoglobin levels further supported the diagnosis for rhabdomyolysis. It is a significant piece of information to alert the emergency physician to the possibility of such significant muscle damage and raised liver function tests in a case of streptococcal pharyngitis.

Correspondence to: Dr. Sadaf Sheikh, Department of Emergency Medicine, Sultan Qaboos University Hospital, Muscat, Oman

E-mail:sheikh.sadaf@gmail.com

Received: October 07, 2019; Revised: March 03, 2020;

Accepted: March 10, 2020

DOI: https://doi.org/10.29271/jcpsp.2021.06.732

\section{CASE REPORT}

A previously healthy, 18-year gentleman with a history of fever and sore throat for 10 days, managed at a tertiary care hospital, was referred to our hospital due to worsening renal functions with the laboratory report showing serum creatinine of 8.1 $\mathrm{mg} / \mathrm{dL}$ (normal: $0.7-1.2 \mathrm{mg} / \mathrm{dL}$ ). According to the patient, he developed fever 10 days back, which was high grade, with chills, was continuous, and partially relieved with oral acetaminophen. Patient complained of severe body aches. There were no urinary symptoms, cough, abdominal pain or headache. The patient was initially managed as an upper respiratory tract infection and was started on amoxicillin. After 5 days course of antibiotics, fever remained unsettled and the patient developed nausea and vomiting with decreased appetite and proximal muscle pain. The patient was hospitalised and initial laboratory workup revealed elevated total leukocyte count (TLC) of $25.2 \times 10^{9} / \mathrm{L}$ (normal: $4.3-10.8 \times$ $\left.10^{9} / \mathrm{L}\right)$, elevated liver enzymes with aspartate aminotransferase (AST) of $1862 \mathrm{U} / \mathrm{L}$, alanine aminotransferase (ALT) of $1003 \mathrm{U} / \mathrm{L}$ and serum creatinine of $7.2 \mathrm{mg} / \mathrm{dL}$. Malarial parasite smear and ICT malaria antigen were negative. The patient was empirically started on intravenous ceftriaxone 2 grams once daily. The patient's renal functions worsened further and he was referred to our hospital. In addition to prior symptoms, patient now complained of worsening muscle pain and difficulty in getting up from sitting position. There was no history of trauma, no related prior family history, no involvement in contact sports or no prior medication history.

On examination, he was afebrile, with normal blood pressure, normal pulse rate and normal oxygen saturation on pulse 
oximeter with normal respiratory rate. General physical examination was unremarkable except for hyperemic throat. There was no jaundice, anemia, cyanosis, clubbing, palpable lymph nodes, edema, or enlarged thyroid. The chest was clear with normal vesicular breathing. Cardiovascular examination was unremarkable except for the tapping apex beat. There was no audible murmur and examination revealed normal first and second heart sounds. The abdominal and genitourinary examination was unremarkable. The patient was catheterised at the outside setting with the urine bag containing about $400 \mathrm{ml}$ of urine with gross hematuria.

Initial workup repeated at our hospital revealed elevated TLC of $28.3 \times 10^{9} / \mathrm{L}$, with neutrophilic shift of $87.9 \%$, normal hemoglobin, normal platelet count, blood urea nitrogen of $84 \mathrm{mg} / \mathrm{dl}$, creatinine of $9.3 \mathrm{mg} / \mathrm{dl}$, serum potassium of $7.2 \mathrm{mmol} / \mathrm{l}$, serum magnesium of $3.1 \mathrm{mg} / \mathrm{dl}$, and serum calcium of $8.8 \mathrm{mg} / \mathrm{dl}$. Liver function tests revealed AST of $994 \mathrm{U} / \mathrm{L}, \mathrm{ALT}$ of $1529=\mathrm{U} / \mathrm{L}$, normal total bilirubin, direct bilirubin, indirect bilirubin, gamma glutamyl transferase (GGT), normal alkaline phosphatase, and prothrombin time of 10.6 seconds. CPK was $111856 \mathrm{IU} / \mathrm{L}$ (normal: 46-171 IU/L). Urinalysis was positive for hemoglobin $5+$, protein 2+, leukocyte esterase 3+, RBC >20/HPF, and leucocytes, 12/HPF. Coombs, HEVIgM, HAVIgM, HBsAg, anti-HCVantibody, malarial parasite smear and ICT malaria antigen were negative. Additional workup revealed elevated ASO titres of $400 \mathrm{lU} / \mathrm{ml}$ (normal <200 IU/ml), and erythrocyte sedimentation rate (ESR) of $49 \mathrm{~mm} / 1 \mathrm{st} \mathrm{hr}$ (normal: 0-15). Antinuclear antibody (ANA) was positive with the homogenous pattern and procalcitonin was $0.52 \mathrm{ng} / \mathrm{ml}$ (normal <0.5). Blood cultures grew no organisms.

Management was started on the lines of non-traumatic rhabdomyolysis with aggressive intravenous fluids resuscitation and sodium bicarbonate infusion. Potassium lowering therapy was given twice, with rectal polystyrene sodium to correct hyperkalemia. Potassium repeated after 4 hours was 6.3 $\mathrm{mmol} / \mathrm{l}$. The patient was planned for hemodialysis due to low urinary out and refractory hyperkalemia. Two sessions of hemodialysis were performed. Due to the history of prior hospitalisation, intravenous piperacillin / tazobactam was started empirically at renal adjusted dose due to presumed sepsis. Laterintravenous vancomycin was also added. The patient's laboratory work-up on the fourth day of admission showed marked improvement in CPK level, i.e., $7904 \mathrm{IU} / \mathrm{L}$. TLC improved to $19.7 \times 10^{9} / \mathrm{L}$, blood urea nitrogen improved to $43 \mathrm{mg} / \mathrm{dl}$ and serum creatinine to $4.9 \mathrm{mg} / \mathrm{dl}$. The patient also improved symptomatically. Fever, nausea and vomiting were improved. The patient's family was counselled for the continuous need of inpatient care with the possible need of dialysis during hospital stay and additional need for intravenous hydration; but due to extreme financial constraints, the patient refused further workup and left against medical advice on 4th day of admission.

\section{DISCUSSION}

Group A $\beta$-hemolytic streptococci are the most frequently isolated pathogens in acute pharyngitis. ${ }^{1}$ Streptococcal infec- tion causing acute rhabdomyolysis is a rare entity with few case reports published in the literature. Pathophysiology inquiring causal relationship is yet not well known and revolves around direct invasion or toxin damage with sepsis as a trigger in this case. However, no exact toxin has been isolated. ${ }^{2}$ _other triggers could be alcohol ingestion, smoking, compression injury and generalised seizures. Whatever the mechanism of rhabdomyolysis in such invasive infections, released myoglobin itself is a direct renal toxin causing cortical ischemia and results in the rise of CPK from $261 \mathrm{IU} / \mathrm{L}$ to $>50,000 \mathrm{IU} / \mathrm{L}^{3}{ }^{3}$

Our case manifested relatively mild pharyngitis with streptococcal infection which got resolved in a week with unsettling myalgias. There was no history of any muscle injury. We found very highlevels of CPK, which have not been reported in the literature. We feel it is important to alert other physicians to the possibility that $\beta$-haemolytic streptococca/infections can be associated with significant muscle destruction and liver damage. Our patient underwent hemodialysis twice to help normalise the creatinine and help the patient to make adequate urine output.

Elevated transaminases is a rare complication of $\beta$-haemolytic streptococci-related pharyngitis. Although the exact prevalence and pathophysiological mechanisms are undetermined; direct bacterial injury, toxicity and immunologic mediation have been proposed. ${ }^{4}$ Liver biopsies have shown granulocytic infiltration of the portal areas and hepatocytic degeneration. In most patients, the prognosis is excellent. ${ }^{5}$

As a food for thought and to avoid morbidity and mortality from $\beta$-haemolytic streptococcal infections, physicians should be aware of rhabdomyolysis in patients presenting with pharyngitis, skin infections or pneumonia with myalgia and consider checking CPKatadmission, which could help managing the critically ill patients.

\section{PATIENT'S CONSENT:}

Verbal consent was obtained from the patient to publish the data concerning this case.

\section{CONFLICT OF INTEREST:}

None to declare.

\section{AUTHORS' CONTRIBUTION:}

SS, UJ, MAB: Significantly contributed in drafting and editing the work.

\section{REFERENCES}

1. Naik TB, Nadagir SD, Biradar A. Prevalence of betahemolytic streptococci groups A, C, and G in patients with acute pharyngitis. J Lab Physicians 2016; 8(1):45-9.

2. Gabow PA, Kaehny WD, Kelleher SP. The spectrum of rhabdomyolysis. Med (Baltimore) 1982; 61(3):141-52.

3. Iyasere CA, Simmons LH, Fintelmann FJ, Dighe AS. Case records of the massachusetts general hospital. Case 38-2014. An 87-year-old man with sore throat, hoarseness, fatigue, and dyspnea. N Engl J Med 2014; 371(24):2321-7. 
4. Ramos-Paesa C, Huguet-Embún L, Arenas-Miquelez Al, De Los Mozos-Ruano A. Fever of unknown origin and rhabdomyolysis in an immigrant from Africa. Enferm Infecc Microbiol Clin 2019; S0213-005X (19)30302-7.
5. Fereshte S, HamidReza N, Seddigheh SE, Masoumeh G. A complicated course of acute viral induced pharyngitis, icteric hepatitis, acalculous cholecystitis and skin rash. Case Rep Med 2016; 2016:6796094. 